\title{
AN INTRINSIC CHARACTERISATION OF POLYHOMOGENEOUS LAGRANGIAN DISTRIBUTIONS
}

\author{
M. S. JOSHI
}

(Communicated by Jeffrey B. Rauch)

\begin{abstract}
The purpose of this paper is to present a method of characterising polyhomogeneous Lagrangian distributions via testing by pseudo-differential operators. The concept of a radial operator for a Lagrangian submanifold is introduced, and polyhomogeneous Lagrangian distributions are shown to be the only Lagrangian distributions which are eigenfunctions at the top order for these operators.
\end{abstract}

\section{INTRODUCTION}

In the study of linear partial differential equations using microlocal analysis, a powerful technique is to transform a problem using symplectic geometry to a more easily understood model problem. This approach relies on using the calculus of Lagrangian distributions which was introduced by Hörmander in [2]. These spaces can be defined in two different ways: the first is geometric and a priori invariant whereas the second is constructive but one has to work hard to show invariance. One advantage of the constructive definition is that it distinguishes the "classical" distributions, the symbols of which have asymptotic expansions in homogeneous terms whereas the geometric definition yields general symbols with $L^{\infty}$ bounds. In some circumstances, for example in the study of propagation of singularities for non-linear wave equations, there are no constructive proofs that the distributions arising are Lagrangian and hence the question of polyhomogeneity is unresolved. Our purpose, in this paper, is to give a geometric characterisation of the polyhomogeneous distributions by introducing the "radial operator" of a Lagrangian submanifold. These operators were used in [4], [5] to understand the more complicated issue of polyhomogeneity for distributions associated to two intersecting Lagrangian submanifolds which allowed the construction of a Fourier integral operator invariant calculus of paired Lagrangian distributions with no error at the intersection.

To define a Lagrangian distribution in a geometric manner, one requires that the distribution remains in a fixed Sobolev space under repeated application of first order pseudo-differential operators which are characteristic on a given fixed

Received by the editors September 20, 1995 and, in revised form, November 14, 1995.

1991 Mathematics Subject Classification. Primary 58G15.

Key words and phrases. Lagrangian, polyhomogeneity, partial differential equations.

This research forms part of my thesis research carried out at the Massachusetts Institute of Technology under the supervision of R.B. Melrose, and I would like to thank him for his guidance and advice. 
Lagrangian submanifold, $\Lambda$. This is equivalent to the definition originally made by Hörmander in [2] that a distribution is Lagrangian of order $m$ with respect to a submanifold, $\Lambda$, if it can locally be represented as

$$
\int e^{i \phi(x, \theta)} a(x, \theta) d \theta
$$

with $\phi$ a non-degenerate phase function such that $\Lambda=\left\{\left(x, \phi_{x}^{\prime}\right): \phi_{\theta}^{\prime}=0\right\}$ and $a$ is in the symbol class $S_{1,0}^{m+\frac{n}{4}-\frac{N}{2}}\left(\mathbb{R}_{x}^{n} \times \mathbb{R}_{\theta}^{N}\right)$, that is, $a$ satisfies estimates of the form

$$
\left|D_{x}^{\alpha} D_{\theta}^{\beta} a(x, \theta)\right| \leq C_{\alpha, \beta, K}(1+|\theta|)^{m-|\beta|}, \quad x \in K, K \text { compact. }
$$

(see [3], Chapter 25)

We say that the symbol $a$ is polyhomogeneous (of step one) or classical if it has an asymptotic expansion in homogeneous terms, i.e. there exist

$$
a_{\mu-j}(x, \theta) \in C^{\infty}\left(\mathbb{R}_{x}^{n} \times\left(\mathbb{R}_{\theta}^{N}-\{0\}\right)\right)
$$

homogeneous of degree $\mu-j$ in $\theta$ such that

$$
a-\sum_{j<N} a_{\mu-j} \in S_{1,0}^{\Re \mu-N}, \text { for }|\theta| \text { large. }
$$

A Lagrangian distribution is said to be polyhomogeneous if the symbol $a$ in the representation (1.1) is polyhomogeneous. We present a geometric way to distinguish these polyhomogeneous distributions by using the fact that at the top level they are eigenvectors of a special class of operators associated to the Lagrangian submanifold. We shall say a classical, first order pseudo-differential operator is "radial" for a conic Lagrangian submanifold, $\Lambda$, if on $\Lambda$, it is characteristic, has sub-principal symbol equal to $\frac{3 n}{4}$ and has bicharacteristic flow equal to $i^{-1}$ times the natural radial vector field on $\Lambda$. These first order pseudo-differential operators generalise the concept of a radial vector field for a submanifold introduced by Melrose in [6], and we show that they always exist. We prove that a distribution is polyhomogeneous of order $\mu$ for a Lagrangian $\Lambda$ if and only if, for any radial operator $R$ for $\Lambda$,

$$
\left(\prod_{j=0}^{N-1}(R+\mu-j)\right) u \in I^{\Re \mu-N}(\Lambda), \text { for all } N .
$$

\section{The MODEL CASE}

First, we establish our results for the simplest case, and later we deduce that they remain true in general. So in this section, we take

$$
\Lambda=N^{*}\{x=0\} \subset T^{*}\left(\mathbb{R}^{n}\right)-0
$$

and work with the radial operator $x \frac{\partial}{\partial x}$. Now, $u \in I_{p h g}^{\mu+n / 4}(\Lambda)$ if and only if

$$
u=\int e^{i x \cdot \xi} a(\xi) d \xi+C^{\infty}
$$

and $a$ is a symbol with polyhomogeneous expansion $\sum a_{\mu-j}$. Euler's relation tells us that

$$
\left(\xi \frac{\partial}{\partial \xi}-(\mu-j)\right) a_{\mu-j}(\xi)=0
$$


and thus we deduce that

$$
\prod_{j=0}^{N-1}\left(\xi \frac{\partial}{\partial \xi}-(\mu-j)\right) a(\xi) \in S^{\Re \mu-N} .
$$

In fact, this relation is equivalent to polyhomogeneity.

Proposition 2.1. A symbol a is polyhomogeneous of order $\mu$ if and only if for all $N$

$$
\prod_{j=0}^{N-1}\left(\xi \frac{\partial}{\partial \xi}-(\mu-j)\right) a(\xi) \in S^{\Re \mu-N} .
$$

Proof. We have already established the proposition in one direction; the other direction is a little harder. If $a=a_{\mu}+e$ with $e \in S^{\Re \mu-1}$, then $\lambda^{-\mu} a(\lambda \xi)=$ $a_{\mu}(\xi)+O\left(\lambda^{-1}\right)$, and so in the limit as $\lambda \rightarrow \infty$ we obtain $a_{\mu}(\xi)$. We use this idea to find $a_{\mu}$ given (2.2). Changing to polar coordinates $\xi=\lambda \omega$ we have

$$
\left(\lambda \frac{\partial}{\partial \lambda}-\mu\right) a(\lambda, \omega)=O\left(\lambda^{\Re \mu-1}\right)
$$

or

$$
\left(\frac{\partial}{\partial \lambda}\left(\lambda^{-\mu} a(\lambda, \omega)\right)\right)=O\left(\lambda^{-2}\right)
$$

and so integrating out to infinity, we deduce that $a_{\mu}(\xi)=\lim _{\lambda \rightarrow \infty} \lambda^{-\mu} a(\lambda \xi)$ converges, and it is immediate that it is homogeneous of degree $\mu$. Differentiation in $\omega$ and $\lambda \frac{\partial}{\partial \lambda}$ commute with this argument, and so, integrating $\lambda^{-\mu}\left(a-a_{\mu}\right)$ in from $\infty$, we deduce that $a-a_{\mu} \in S^{\Re \mu-1}$. It is immediate that

$$
\left|\left(\lambda \frac{\partial}{\partial \lambda}-\mu\right) \prod_{j=1}^{N-1}\left(\lambda \frac{\partial}{\partial \lambda}-(\mu-j)\right)\left(a-a_{\mu}\right)(\lambda, \omega)\right| \leq C|\lambda|^{\Re \mu-N},
$$

so, considering

$$
\left|\frac{\partial}{\partial \lambda}\left(\lambda^{-\mu} \prod_{j=1}^{N-1}\left(\lambda \frac{\partial}{\partial \lambda}-(\mu-j)\right)\left(a-a_{\mu}\right)(\lambda, \omega)\right)\right|
$$

as $\lambda \rightarrow \infty$ and integrating, we deduce that

$$
\left|\prod_{j=1}^{N-1}\left(\lambda \frac{\partial}{\partial \lambda}-(\mu-j)\right)\left(a-a_{\mu}\right)(\lambda, \omega)\right| \leq C|\lambda|^{\Re \mu-N}
$$

and the derivative estimates follow as before. Thus we have shown that $a-a_{\mu}$ satisfies our original hypothesis (with orders suitably shifted), and so the result follows by induction.

Taking the Fourier transform of the statement of this proposition, we obtain

Corollary 2.1. An element $u$ of $I(\Lambda)$ is polyhomogeneous of order $\mu+\frac{n}{4}$ if and only if for all $N$

$$
\prod_{j=0}^{N-1}\left(x \frac{\partial}{\partial x}+n+\mu-j\right) u \in I^{\Re \mu+\frac{n}{4}-N}(\Lambda) .
$$


The $n / 4$ is an artifact of the way Lagrangian orders are defined - see (1.1).

Thus we now have a characterisation; the problem is that the operator $x \frac{\partial}{\partial x}$ is not even coordinate invariant whereas we want an operator which is invariant under conjugation by classical Fourier integral operators associated to symplectomorphisms which preserve the Lagrangian submanifold. Full invariance turns out to be impossible, but we can obtain invariance up to harmless terms, and so we work with a class of radial operators rather than a single one.

\section{General Lagrangian submanifolds}

In this section, we will study general conic Lagrangian submanifolds of the cotangent bundle of a smooth manifold $X$ of dimension $n$. We will work with half-densities, as then the sub-principal symbol of a pseudo-differential operator is well-defined. (See for example [1].) To define a radial operator for a general Lagrangian submanifold, we can either abstract the important symbolic properties and use them as a definition, or we can use the mapping properties on polyhomogeneous Lagrangian distributions. We do both and show they are equivalent.

Recall that a vector space, $V$, carries a natural vector field which generates the radial action:

$$
(\lambda, v) \mapsto \lambda v, \lambda \in \mathbb{R}_{+}, v \in V .
$$

In local coordinates, this takes the form $x \frac{\partial}{\partial x}$. So since the cotangent bundle is a vector bundle, we have a natural radial vector field which takes the form $\xi \frac{\partial}{\partial \xi}$ if $\xi$ are dual coordinates to local coordinates on $M$.

Definition 3.1. A radial operator for an embedded, conic, Lagrangian submanifold $\Lambda$ of $T^{*}(X)-0$ is a properly supported, classical pseudo-differential operator, $R$, of first order such that

(1) the principal symbol of $R$ vanishes on $\Lambda$,

(2) the sub-principal symbol of $R$ equals $n / 4$ on $\Lambda$,

(3) the bicharacteristic field of $R, H_{R}$, on $\Lambda$ is equal to the radial vector field multiplied by $i^{-1}$.

Note that with these normalisations, $x \frac{\partial}{\partial x}+\frac{3 n}{4}$ will be a radial operator for $N^{*}\{x=0\}$ in $\mathbb{R}^{n}$.

Theorem 3.1. Given a conic Lagrangian submanifold, $\Lambda$, a first order classical pseudo-differential operator, $P$, is radial for $\Lambda$ if and only if

$$
P+\mu: I_{p h g}^{\mu}\left(\Lambda, \Omega^{\frac{1}{2}}\right) \rightarrow I_{p h g}^{\mu-1}\left(\Lambda, \Omega^{\frac{1}{2}}\right) \text { for all } \mu .
$$

The difference of two radial operators will always induce a map

$$
P_{1}-P_{2}: I_{p h g}^{\mu}\left(\Lambda, \Omega^{\frac{1}{2}}\right) \rightarrow I_{p h g}^{\mu-1}\left(\Lambda, \Omega^{\frac{1}{2}}\right) .
$$

Proof. The second statement follows trivially from the first. To prove the theorem we compute the principal symbol of $(R+\mu) u$ for $u \in I_{p h g}^{\mu}(\Lambda)$. The principal symbol as an element of $I_{p h g}^{\mu+1}(\Lambda)$ is $\sigma_{1}(R) \sigma_{\mu}(u)$ on $\Lambda$, so this can vanish for all $u$ if and only if $\sigma_{1}(R)$ is zero on $\Lambda$, which is our first property. The principal symbol of $(R+\mu) u$ at one level lower will now be

$$
\frac{1}{i} \mathcal{L}_{H_{R}} \sigma_{\mu}(u)+\left(\sigma_{\mathrm{sub}}(R)+\mu\right) \sigma_{\mu}(u)
$$


where $\mathcal{L}_{H_{R}}$ denotes the Lie derivative acting on 1/2-densities. Thus we need (3.1) to vanish for all $\mu$. Since this is true for any polyhomogeneous $u$, this tells us that if $\sigma_{\mu}(u)$ vanishes along a ray so does $\mathcal{L}_{H_{R}} \sigma_{\mu}(u)$, and hence that $H_{R}$ is tangent to rays, which means that it is a multiple of the radial vector field $\rho$.

Now we can pick local coordinates $x$ on the manifold such that the dual coordinates $\xi$ parametrise $\Lambda$, and then $|d \xi|^{\frac{1}{2}}$ is a non-vanishing $1 / 2$-density on $\Lambda$. In these coordinates the radial vector field is $\xi \frac{\partial}{\partial \xi}$. Writing $H_{R}=\phi \rho$ we obtain, using Euler's relation, that

$$
\begin{aligned}
\frac{1}{i} \phi(\xi)\left(\mu-\frac{n}{4}\right) a_{\mu-\frac{n}{4}}+ & \frac{1}{i} \phi(\xi) \frac{n}{2} a_{\mu-\frac{n}{4}} \\
& +\frac{1}{2 i} \sum \xi_{j} \frac{\partial \phi}{\partial \xi_{j}} a_{\mu-\frac{n}{4}}+\mu a_{\mu-\frac{n}{4}}+\sigma_{\mathrm{sub}}(R) a_{\mu-\frac{n}{4}}=0
\end{aligned}
$$

where $a_{\mu-\frac{n}{4}}(\xi)|d \xi|^{\frac{1}{2}}$ is the principal symbol of $u$. From this, we deduce

$$
\mu\left(\frac{\phi(\xi)}{i}+1\right)+\left(-\frac{1}{i} \frac{n}{4} \phi(\xi)+\frac{n}{2 i} \phi(\xi)+\frac{1}{2 i} \sum \xi_{j} \frac{\partial \phi}{\partial \xi_{j}}+\sigma_{\mathrm{sub}}(R)\right)=0 .
$$

This is true for all $\mu$, so $\phi(\xi)=-i$ and $\sigma_{\text {sub }}(R)=\frac{n}{4}$, which establishes that $R$ is radial.

To see that a radial operator has the correct mapping properties, observe that property (1) implies that $(R+\mu) u$ is in $I_{p h g}^{\mu}\left(\Lambda, \Omega^{\frac{1}{2}}\right)$, and then the principal symbol will be given by (3.1). It now follows from Euler's relation that

$$
\frac{1}{i} \mathcal{L}_{H_{R}} \sigma_{\mu}(u)=-(\mu+n / 4) \sigma_{\mu}(u),
$$

and hence the result follows from property (2).

Having shown the equivalence of two definitions of a radial operator, we need to show that they do in fact exist. Locally, a Lagrangian submanifold can be parametrised by a phase function of the form $\phi(x, \xi)=x . \xi-H(\xi)$ in some system of local coordinates $x$ on $X$-for a proof of this see [2]. A pseudo-differential operator with total symbol equal to $i \phi+\frac{3 n}{4}$ smoothed off at $\xi=0$ will be a radial operator for $\Lambda$ within the coordinate system - to see this, just apply the operator to a polyhomogeneous distribution and integrate by parts. So the local existence is clear. To get global existence, one can patch together these local operators via a micro-local partition of unity to obtain an operator that has the correct principal symbol and bicharacteristic flow on the submanifold. It is not clear that this operator will have the correct sub-principal symbol, but this can always be adjusted to the requisite value by subtracting a pseudo-differential operator of zeroth order. Hence we have

Proposition 3.1. The class of radial operators associated to an embedded, conic, Lagrangian submanifold is always non-empty.

So having found our analogue of $x \frac{\partial}{\partial x}$ for a general Lagrangian submanifold, we wish to prove the analogue of Corollary 2.1. 
Theorem 3.2. An element $u$ of $I^{\Re \mu}(\Lambda)$ is polyhomogeneous of order $\mu$ if and only if for some (and hence any) radial operator $R$ associated to $\Lambda$,

$$
\left(\prod_{j=0}^{N-1}(R+\mu-j)\right) u \in I^{\Re \mu-N}(\Lambda) \text {, for all } N .
$$

Proof. Micro-locally, we can always map a Lagrangian submanifold to the model $N^{*}\{x=0\}$ by a symplectomorphism (see [3], Chap. 21), so if we pick an associated elliptic zeroth order Fourier integral operator and conjugate, it is enough to prove this theorem for the model. So our proof reduces to showing that if (3.4) holds for one radial operator then it necessarily holds for $V=x \frac{\partial}{\partial x}+\frac{3 n}{4}$. To prove this we require a lemma:

Lemma 3.1. Let $Q$ be a classical pseudo-differential operator which maps

$$
I^{m}\left(N^{*}\{x=0\}\right) \quad \text { to } \quad I^{m-k}\left(N^{*}\{x=0\}\right) .
$$

Then $[V, Q]-k Q$ maps $I^{m}\left(N^{*}\{x=0\}\right)$ to $I^{m-k-1}\left(N^{*}\{x=0\}\right)$.

Proof. Suppose $Q$ is of order $l$ and has total symbol with asymptotic expansion $\sum q_{l-j}$. Then on application to a conormal distribution with asymptotic expansion $\sum b_{m-j}$ one obtains the asymptotic expansion

$$
\sum_{j+r+|\alpha|=s} C_{\alpha} D_{x}^{\alpha} q_{l-j}(0, \xi) D_{\xi}^{\alpha} b_{m-r}(\xi) .
$$

As $b_{m-j}$ is arbitrary, for our hypothesis to be fulfilled we will need $q_{l-j}(x, \xi)$ to vanish to order $l-j+k$ at $x=0$, and the same computation shows that this is in fact equivalent to the mapping property. Thus, by Taylor's theorem, we can write

$$
q_{l-j}=q_{l-j}^{\prime}+r_{l-j}
$$

where $q_{l-j}^{\prime}$ is a homogeneous polynomial of degree $k+l-j$ in $x$ and $r_{l-j}$ vanishes to order $k+l+1-j$ at $x=0$. The $j^{\text {th }}$ term in the total symbol of $[V, Q]$ will then be

$$
\left(x \frac{\partial}{\partial x}-\xi \frac{\partial}{\partial \xi}\right) q_{l-j}^{\prime}+\left(x \frac{\partial}{\partial x}-\xi \frac{\partial}{\partial \xi}\right) r_{l-j}^{\prime} .
$$

The bihomogeneity of $q_{l-j}^{\prime}$ means that the first term will be $k q_{l-j}^{\prime}$, and thus $[V, Q]-$ $k Q$ will have the requisite mapping property as the $r_{l-j}^{\prime}$ terms will retain the needed vanishing properties.

So we know that our hypothesis holds for $V+P$ where $P$ is a first order, classical pseudo-differential operator which maps $I^{m}(\Lambda)$ to $I^{m-1}(\Lambda)$ for each $m$. It is clear that $(V+\mu+P) u \in I_{p h g}^{\Re \mu-1}$ if and only if $(V+\mu) u \in I_{p h g}^{\Re \mu-1}$, so our result is true for $N=1$. We prove the general case by induction: suppose that

$$
\prod_{j=0}^{k-1}(V+(\mu-j)) u \in I^{\Re \mu-k}(\Lambda), \quad k \leq N-1,
$$

and we have from our hypothesis that

$$
\prod_{j=0}^{N}(V+(\mu-j)+P) u \in I^{\Re \mu-N-1}(\Lambda) .
$$


We commute the operators $P$ to the left and, applying our lemma repeatedly, obtain

$$
\sum_{k=0}^{N} T_{k} \prod_{j=0}^{N-k}(V+\mu-j) u \in I^{\Re \mu-N-1}(\Lambda),
$$

where $T_{k}$ denotes a classical pseudo-differential operator which induces a map from $I^{m}(\Lambda)$ to $I^{m-k}(\Lambda)$. Thus, invoking the inductive hypothesis, the result follows.

\section{REFERENCES}

[1] J.J. Duistermaat and L. Hörmander, Fourier Integral Operators II. Acta Mathematicae 128 (1972), 183-269. MR 52:9300

[2] L. Hörmander, Fourier Integral Operators I, Acta Mathematicae 127 (1971), 79-183. MR 52:9299

[3] L. Hörmander, Analysis of Linear Partial Differential Operators, Vol. 1 to 4, Springer Verlag, Berlin, 1983, 1985. MR 85g:35002a,b; MR 87d:35002a,b

[4] M.S. Joshi, A Precise Calculus of Paired Lagrangian Distributions, M.I.T. thesis, 1994.

[5] M.S. Joshi, A Symbolic Contruction of the Forward Fundamental Solution of the Wave Operator, preprint

[6] R.B. Melrose, Differential Analysis on Manifolds with Corners, forthcoming.

Department of Pure Mathematics and Mathematical Statistics, University of CamBridge, 16 Mill Lane, Cambridge CB2 1SB, England, United Kingdom

E-mail address: joshi@pmms.cam.ac.uk 